\title{
Temperature correction in super-resolution reconstruction of thermograms
}

\author{
by W. Jamrozik*, A. Krol* \\ * Silesian Univ. of Technology, 44-100, Konarskiego Str., Gliwice, Poland, wojciech.jamrozik@polsl.pl; \\ adrian.krol@polsl.pl
}

\begin{abstract}
Low spatial resolution is a common drawback of thermographic methods in many real life problems. To overcome this disadvantage software improvements are introduced. In the paper super-resolution methods (SR) are described and compared. Two main factors of such approaches are investigated: the first one is increasing of image spatial resolution and quality, while maintaining the temperature accuracy. The second issue is especially crucial, when absolute temperature values are demanded. Several approaches of temperature correction are considered: global, regional and local. Obtained results prove that proposed correction method can assure that absolute temperature values are consistent on the high resolution image.
\end{abstract}

\section{Introduction}

Imagining in infrared emerged as an important method for assessing condition of various technical object. Continuous observation of machines as well as inspections performed in varying time intervals are a common techniques used by maintenance services. To find places where temperature of objects increases dangerously and to calculate features that can be used for reasoning in automated diagnosing systems high resolution (HR) images are more than desired. Higher spatial resolution offers more pattern information to be analysed. This is especially important when small details have to be observed. In the case of low resolution (LR) images their thermal signatures can be confused to the background emission or noise. Physical constraints, technical difficulties and high cost prevent to infrared camera manufactures to make devices with matrices with a large number of photosensitive elements thus the cheap handhelded cameras produce infrared images with relatively low spatial resolution. Moreover, the IR image quality is also degraded by optical blur, motion blur, environmental noise, etc.

There are hardware and software improvements that are elaborated to overcome this kind difficulties. Among others there are super-resolution (SR) methods, that allow increase of spatial resolution of images. Image superresolution(SR) is a process to combine and aggregate multiple LR images to produce a new HR image. The LR images are typically translated at the subpixel level, and according to that the information available in each LR image can be extracted and combined to obtain a high quality HR image. SR was originally introduced by Tsai and Huang [10]. An observation model was defined for an image sequence consisting of sub-pixel shifts of the same scene. A naive frequency domain method was there elaborated, when the Fourier coefficients of the HR image as a function of the Fourier coefficients of the registered low resolution images.

Nowadays SR is widely applied for regular video information content enhancement [14], surveillance [15, 16], medical diagnosis [17, 18] remote sensing [19, 20], biometric identification recognition [21], surface fault estimation [11].

The main application area of this kind of methods is connected with processing of images acquired in visible light, straight use of some methods for infrared images is difficult. Nevertheless some IR cameras manufacturers are incorporating various SR systems into their products $[1,2,3]$. Among others supersampling and deconvolution approaches are present in IR cameras [1]. Main problem is connected with transformation of pixel values, what in case of thermopixels that are present on infrared thermograms can lead to inconsistent values of temperature in comparison to the original low resolution thermogram. In the case of e.g. RGB images normalization range to [0-255] is possible, in the case of radiometric infrared images this kind of correction is impossible. To make super-resolution approaches valid for practical use in the real word applications correction of temperature values is demanded [1]. Having the knowledge about temperatures in thermopixels in the low resolution images three group of correction methods are introduced: global, regional and local.

\section{Super-resolution}

Super-resolution methods considered in the paper generate high-resoution image based on the series of slightly translated low-resolution images in an iterative way. The first step of SR is the image registration., which is a process of finding correspondence between pixels in all input images. This is needed to project LR pixels into HR grid. Every next iteration of SR method usually causes changes of temperature. Depending on the method used, not only unknown thermopixels but also those present in the input image. Considered methods are well known and widely applied for the visible light images: Robust Superresolution (RS) [5], Iterative Back Projection (IBP) [6] and two-dimensional interpolation (ITP). Unfortunately in presented SR methods know values of pixels were changed so is necessary to correcting values of all pixels based on information from sources images. Temperature correction methods are there 


\subsection{1/qirt.2016.046}

needed to obtain reliable thermograms of high quality. As the reference method bicubic interpolation was applied, where interpolated image was also generated from set of LR input images.

\subsection{Iterative backprojection}

Iterative Back Projection is the classic superesolution method. Due to its low computational complexity it can be used in real-time systems. The aim of this superesolution algorithm is to minimalize the error between the images which are results of consecutive iterations. When the method reaches a desired minimal value of the error or the last iteration it was stopped. After loading the thermal images and transform them into a array of values and next selects the reference thermogram (working thermogram) and its interpolation to the desired resolution. Later all of others thermograms are selected and matching them size to reference array size. For this purpose each element of the array is replaced by the shift estimator. Then the size of the reference array is reduced to the original size so it can be compared with the input thermogram. The difference between the reference array and loaded thermogram is calculated. In the next step dimensions of the reference array are increased again to required size. Temporary array containing the differences between successive thermograms is formed. All temporary arrays is added. After performing these operations for all thermal images in the series the temporary array is added to reference array. Result array is tested for obtaining the stop condition. In the case when this condition is not fulfil the whole process is repeated, assuming that the new reference array is the output array from the previous iteration.

\subsection{Robust Superresolution}

Very similar algorithm to Iterative Back Projection is Robust Superresolution (RS). The working of this algorithm is based on the same mechanism with small difference. The difference is in the way of corrections calculation. In the case of SR it is not the difference between the reference image and the other images in the series. In this algorithm corrections are defined as the median of differences between all thermograms. It gives a big advantage over Iterative Back Projection. Median guarantees the removal of outliers in measurements. There is a possibility that during the data collection will be a sudden change of temperature in observing field. This may be caused by the entrance some person in the field of view of the camera or accidental reflection of infrared radiation from other sources. The value of correction obtained in Iterative Back Projection may be very high what is associated to a large change of temperature on the image. In RS method thanks to the use of the median, input image with a many noisy outlier temperatures has little effect on the value of correction.

\section{Temperature correction methods}

Super-resolution algorithms allow to earn a high-resolution (HR) images form low-resolution (LR) source images but temperatures observed on this result image are slightly different in comparison to source images. It is necessary to correct obtained values in order to achieve high certainty of temperatures in the thermopixels generated by SR algorithms. When radiometric accuracy is needed correction is an important procedure in the image processing path [4]. At the stage of preliminary studies three groups of correction methods were distinguished: global, regional and local. In the global approach it has been assumed that one correction factor can be assessed for entire image. In this group methods are easy to implement and use. The main disadvantage of this approach is that also values from original image are changed. In the regional approach all thermopixels in the neighbourhood of the known thermopixel have the same correction factor. Also more sophisticated approach is possible, in which each generated thermopixel has an individual correction factor. The main challenge is to map known values into unknown ones to derive function that allow determination of correction factors. In the performed research methods based on average temperatures deviation between LR and HR images, interpolation methods and difference images are used.

To obtain reliable temperature mapping four simple and not computationally demanding correction methods were elaborated. In all methods the first step is the down sampling of SR image $\left(\boldsymbol{I}_{S R d}\right)$ to the size of LR image. The next step is the calculation of first stage coefficients being the array of ratios between original LR image and down sampled HR image:

$$
\boldsymbol{C}_{1}=\boldsymbol{I}_{L R} / \boldsymbol{I}_{S R d}
$$

The first collected LR image form sequence that was used to generate SR image is used as the reference image. First stage coefficients describe local variations of temperatures in the whole image, and are sensitive to the nonlinearities of temperature distributions on the surface of observed objects. By down sampling of SR image, coefficients can be dynamically calculated for each scene.

The first method is a regional approach (REG), in which the same first stage correction coefficient $\left(C_{1}(x, y)\right)$ is applied to the $\boldsymbol{I}_{S R}$ pixel corresponding to the location on $\boldsymbol{I}_{S R d}$. Additionally same coefficient is applied to the neighbourhood of the $\boldsymbol{I}_{S R}$ pixel in following manner: 


$$
\begin{gathered}
I_{S R C}(m, n)=I_{S R}(m, n) \cdot C_{1}(x, y) \\
I_{S R C}(m+1, n)=I_{S R}(m+1, n) \cdot C_{1}(x, y) \\
I_{S R C}(m+1, n+1)=I_{S R}(m+1, n) \cdot C_{1}(x, y) \\
I_{S R C}(m, n+1)=I_{S R}(m+1, n) \cdot C_{1}(x, y)
\end{gathered}
$$

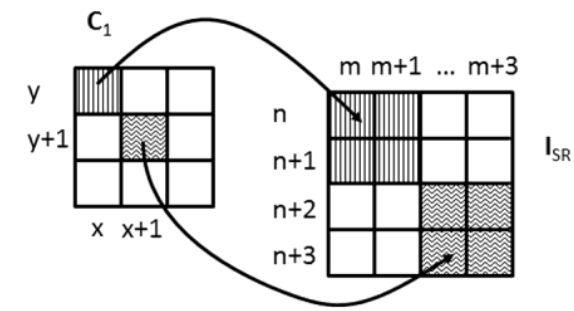

Fig. 1. Idea of correction coefficient application in REG method

For the local method (LOC) slightly different approach was used. In this case the coefficient array $\boldsymbol{C}_{1}$ is interpolated to the size of $\boldsymbol{I}_{S R}$. Bilinear interpolation is used to generate $\boldsymbol{C}_{1 S R}$ array. The $\boldsymbol{I}_{S R C}$ image is than calculated:

$$
\boldsymbol{I}_{S R C}=\boldsymbol{I}_{S R} \cdot \boldsymbol{C}_{1 S R}
$$

The second group of methods contains global approaches. In this case only one correction coefficient is calculated for the whole image. In the GLOB1 method the ratio of total temperature in $\boldsymbol{I}_{L R}$ and $\boldsymbol{I}_{S R d}$ is calculated:

$$
C_{G L O B 1}=\sum \boldsymbol{I}_{L R} / \sum \boldsymbol{I}_{S R d}
$$

Assigned coefficient $C_{G L O B 1}$ is multiplied by each $\boldsymbol{I}_{S R}$ pixel:

$$
\boldsymbol{I}_{S R C}=\boldsymbol{I}_{S R} \cdot C_{G L O B 1},
$$

The GLOB2 method utilizes the mean difference per pixel between $\boldsymbol{I}_{L R}$ and $\boldsymbol{I}_{S R d}$ thermograms.

$$
C_{G L O B 2}=\frac{\sum \boldsymbol{I}_{L R}-\sum \boldsymbol{I}_{S R d}}{N}
$$

where $N$ is the number of pixels in thermogram.

\section{Performance measures}

To find the best correction method from proposed ones two types of quality measures were used. In the first group measures that require reference image are chosen, in the second one several measures describing image properties objectively are selected.

\subsubsection{Energy of Laplacian (LAPE)}

The energy of image second derivative has be widely applied to determine image quality, especially to check if the observed object is in the field of range. The measure is defined as follows [12]:

$$
\phi_{L A P E}=\sum_{(i, j) \in \Omega(x, y)} \Delta I(x, y)^{2},
$$

where $\Delta I$ is the Laplacian of image obtained by convoloving thermogram $I$ with the smoothing Laplacian filter mask.

\subsubsection{Histogram entropy (HISE)}

It is usually expected that image of higher quality will have a higher information content than the degraded one. According to that entropy and histogram range can be both used to determine image (thermogram) quality. The histogram entropy is defined as [13]:

$$
\phi_{H I S E}=-\sum_{k=1}^{L} P_{k} \log \left(P_{k}\right),
$$




\subsection{1/qirt.2016.046}

where $P_{k}$ is the ratio of number of k-th gray level pixels to number of all pixels in image. To compute measure for pixel $\phi_{x, y}$ histogram is calculated for gray levels image $\Omega(x, y)$. To apply this measure to thermograms mapping from temperature range to [0-255] range was applied for fixed minimum and maximum temperatures.

\subsubsection{Natural Image Quality Evaluator (NIQE)}

Natural Image Quality Evaluator (NIQE) [8] is a blind image quality assessment method (IQA). It is a completely blind image quality analyser that uses only deviations observed in natural images. It was originally elaborated for visible light images, but assuming that similar distortions form statistical regularities can be observed in IR images it can be used for thermograms quality evaluation especially in terms of blur and out of focus images.

The NIQE is calculated as a distance between the quality natural scene statistic model and multivariate Gaussian (MVG) fit to features extracted from tested image. Having mean vectors $\left(\boldsymbol{v}_{\mathbf{1}}\right.$ and $\left.\boldsymbol{v}_{\mathbf{2}}\right)$ and covariance matrices of the natural MVG model and the distorted image's MVG model $\left(\boldsymbol{\Sigma}_{\mathbf{1}}\right.$ and $\left.\boldsymbol{\Sigma}_{2}\right)$ the NIQE is defined as follows:

$$
\phi_{N I Q E}=\sqrt{\left(v_{1}-v_{2}\right)^{T}\left(\frac{\Sigma_{1}+\Sigma_{2}}{2}\right)^{-1}\left(v_{1}-v_{2}\right)}
$$

\subsubsection{Information Content Weighted Measures}

Information content weighted measures are calculated by incorporating the idea of information content weighted pooling [9]. Incorporating the Laplacian pyramid transform domain information content weights are calculated. In information content weighted MSE is defined as follows:

$$
\phi_{I W-M S E}=\prod_{j=1}^{M}\left[\frac{\sum_{i} w_{j, i}\left(x_{j, i}-y_{j, i}\right)^{2}}{\sum_{i} w_{j, i}}\right]^{\beta_{j}}
$$

where $x_{i, j}$ and $y_{i, j}$ are the $i$ th transform coefficients at the $j$ th scale, $w_{i, j}$ is the information content weight computed at the corresponding location, $M$ is the number of scales and $\beta_{j}$ is the weight given to the $j$ th scale.

Information content weighted PSNR is expressed by following equation:

$$
\phi_{I W-P S N R}=10 \log _{10}\left(\frac{L^{2}}{\phi_{I W-M S E}}\right)
$$

where $L$ is the image maximum dynamic range.

Information weighted SSIM is an extension of multiscale SSIM. Let $\boldsymbol{x}_{j, i}$ and $\boldsymbol{y}_{j, i}$ be the $i$ th local image patches and $j$ th scale, $N_{j}$ is the number of evaluation windows, and let $c(), s()$ and $l()$ be the contrast, structural and luminance similarities between original and evaluated images. The $j$ th scale $\phi_{I W-S S I M_{j}}$ is defined as:

$$
\phi_{I W-\operatorname{SSIM}_{j}}=\frac{\sum_{i} w_{j, i} c\left(\boldsymbol{x}_{j, i} \boldsymbol{y}_{j, i}\right) s\left(\boldsymbol{x}_{j, i} \boldsymbol{y}_{j, i}\right)}{\sum_{i} w_{j, i}}
$$

for $j=1, \ldots, M-1$ and

$$
\phi_{I W-S S I M_{j}}=\frac{1}{N_{j}} \sum_{i} l\left(\boldsymbol{x}_{j, i}, \boldsymbol{y}_{j, i}\right) c\left(\boldsymbol{x}_{j, i}, \boldsymbol{y}_{j, i}\right) s\left(\boldsymbol{x}_{j, i}, \boldsymbol{y}_{j, i}\right)
$$

for $j=M$. The aggregated $\phi_{I W-S S I M}$ is finally defined as:

$$
\phi_{I W-S S I M}=\prod_{j=1}^{M}\left(\phi_{I W-S S I M}\right)^{\beta_{j}}
$$

\section{Results}

To verify the validity of proposed approach several tests were performed and obtained results were assessed by qualitative and quantitative measures. Two scenes containing different objects were observed were using Jenoptic Infratec HR Head 640 camera. First object (fig. 2a) was a laboratory stand, on which resistance heating wire were placed in different intervals in two directions. The second object (fig. 2b) was a part of power supply system of an industrial tester. It contains power supply unit, relays, fuses, contactors and connection wires. Thermograms of resolution $640 \times 480$ pixels were acquired in sets consisting 5 images. For validation purposes images were decimated, and the resolution was reduced to $320 \times 240$. This operation allows application of reference based measures, where ground through image is required. 
a)
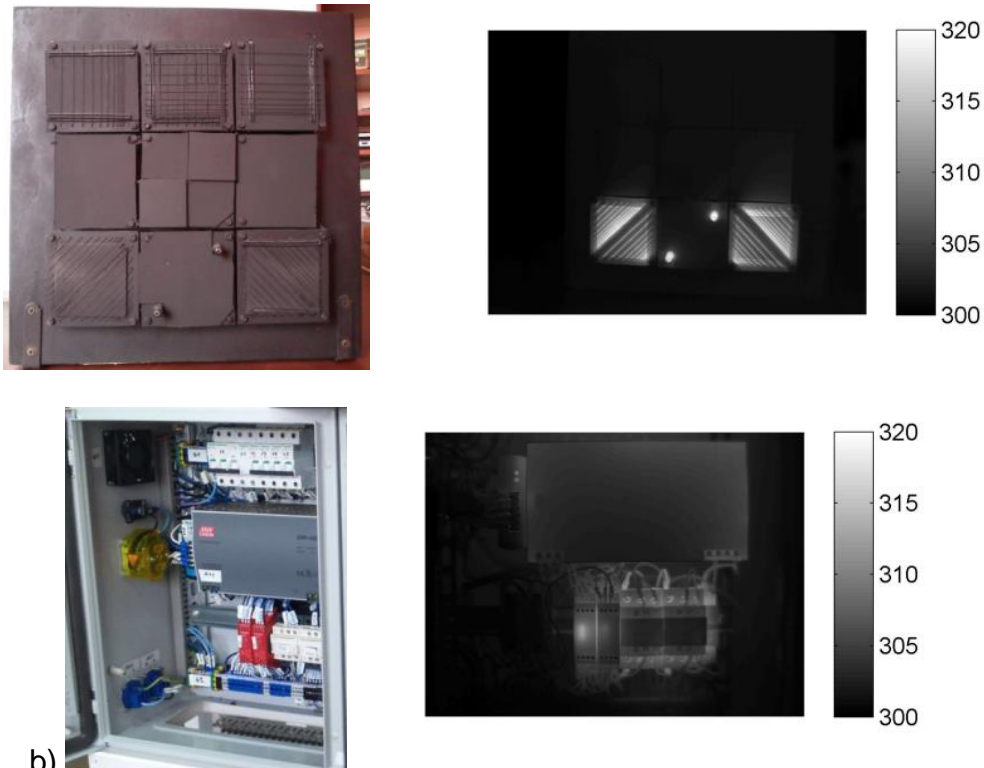

Fig. 2. Test objects: a) test stand, b) electrical equipment

In fig. 3 uncorrected SR thermograms are presented. Subjective evaluation allows to choose best method. Interpolation generates worst results. RS and IBP generates similar results, but RS outputs a slightly sharper HR thermogram. In fig. 4 exemplary correction coefficients arrays calculated by REG and LOC methods are presented. It can be noticed that especially on object edges correction is demanded. Depending on the SR method corrections have various character. When SR thermogram was the result of interpolation, correction is more significant. It is because the $\mathrm{SR}$ image is more blurred as others and to obtain image that is closer to the desired original greater interference in the uncorrected SR image is needed. Analysing only correction arrays the similarity between IBP and RS SR methods can be seen. REG method generates coefficients that preserves more detail, than LOC, when in proposed case details are smoothed. Wire connections as well as internal structure of electric equipment is sharper and edges are clear.

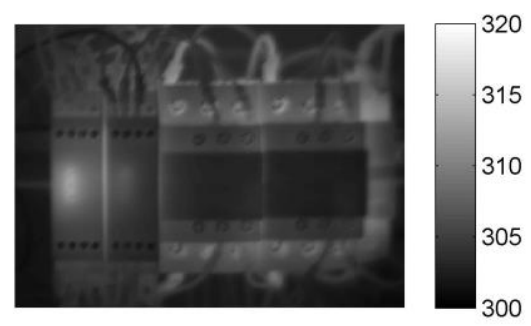

a) IBP

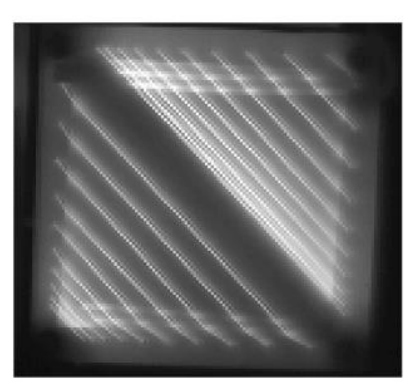

a) IBP

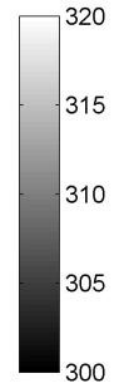

300
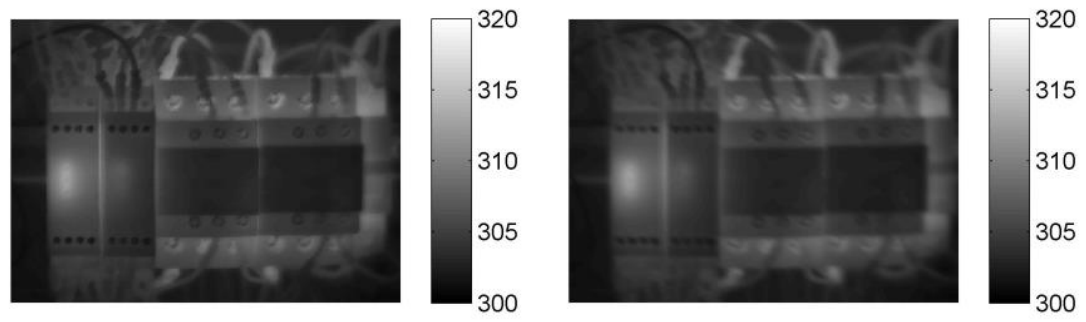

b) RS

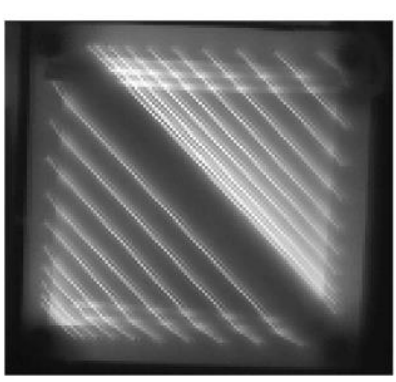

b) RS c) interpolation
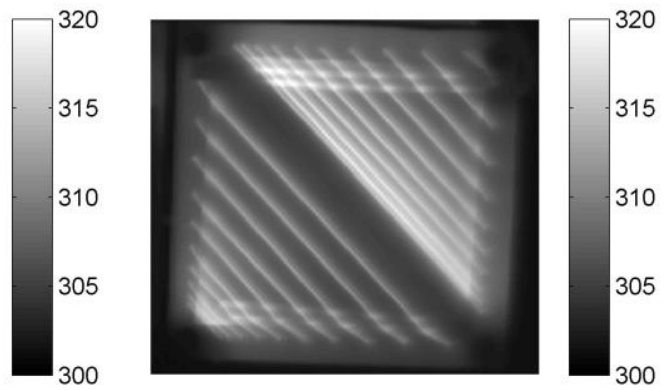

c) interpolation

Fig. 3. SR thermograms without temperature correction 
a) IBP REG

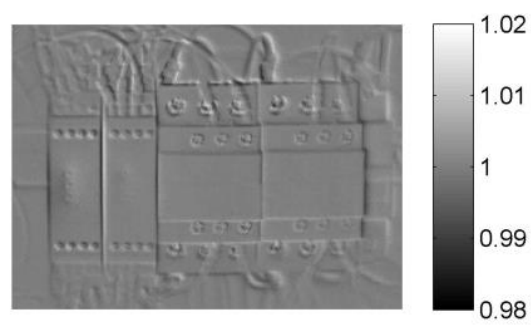

d) IBP LOC

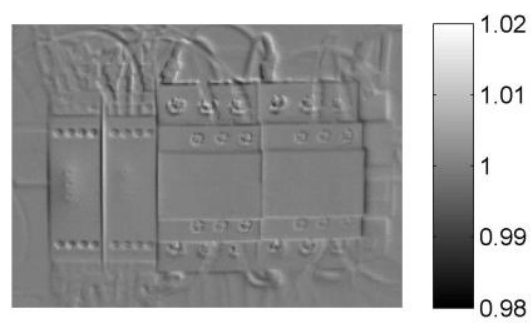

b) RS REG

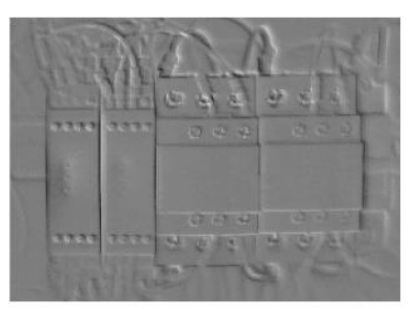

e) RS LOC

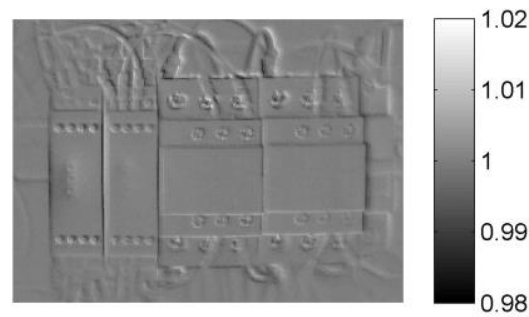

c) Inrerpolation REG
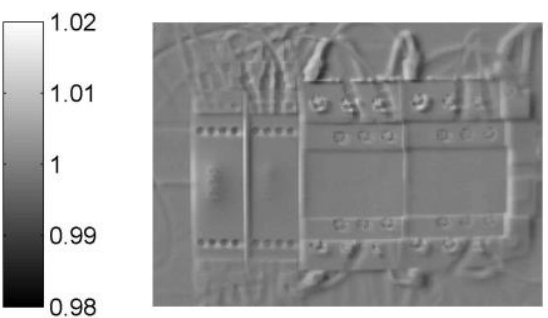

f) Inrerpolation LOC
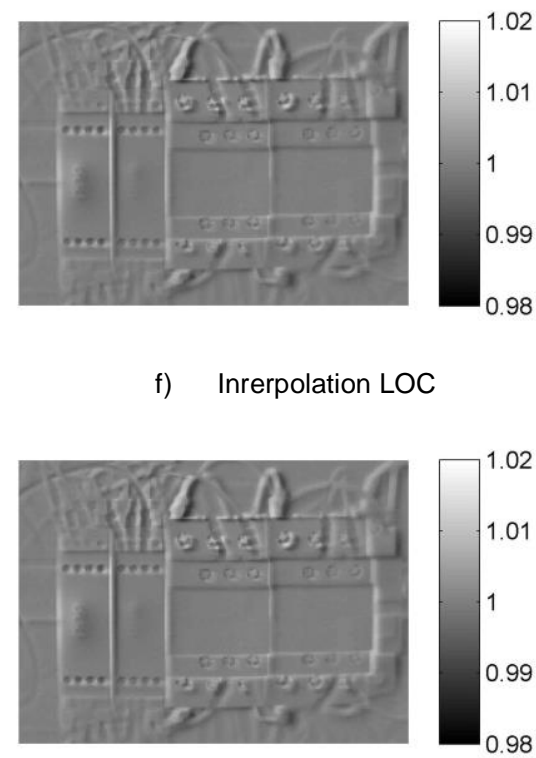

Fig. 4. Correction coefficients maps calculated by REG and LOC methods

a) Original
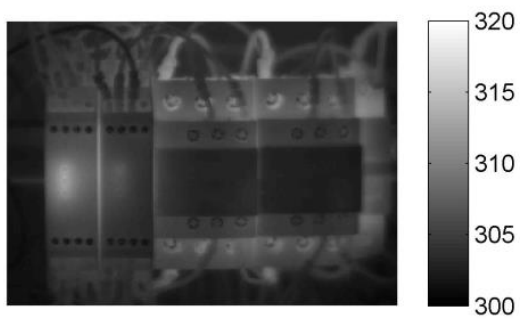

d) IBP GLOB1

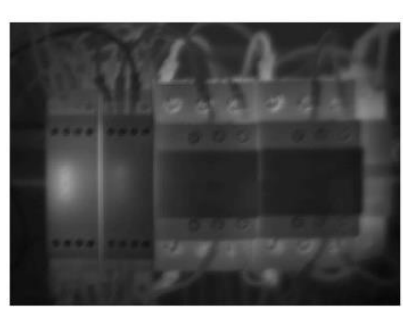

b) IBP REG

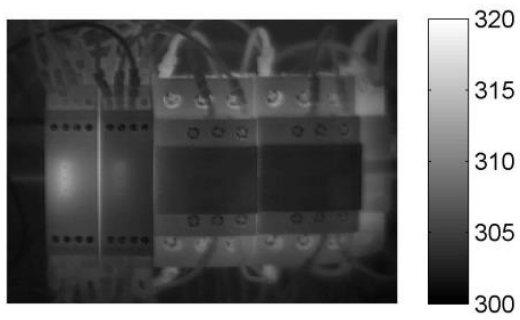

e) IBP GLOB2
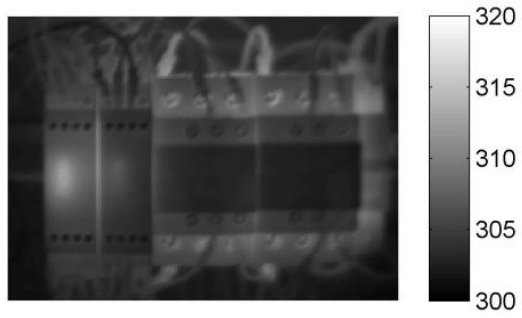

c) IBP LOC

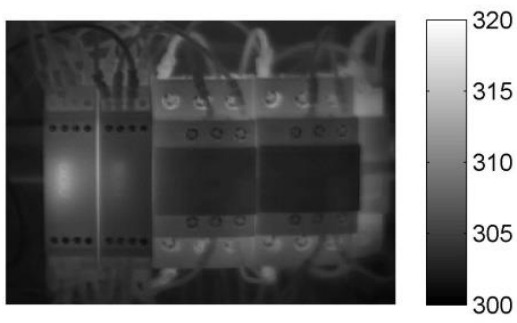

f) IBP without correction

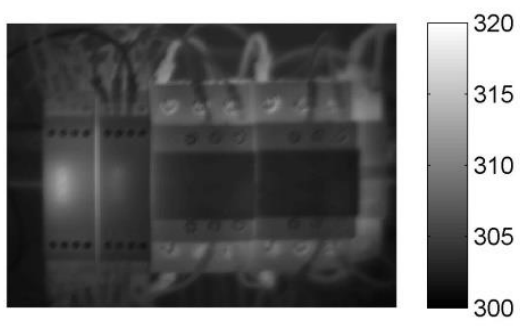

Fig. 5. Comparison of SR correction method 
a) Interpolation REG

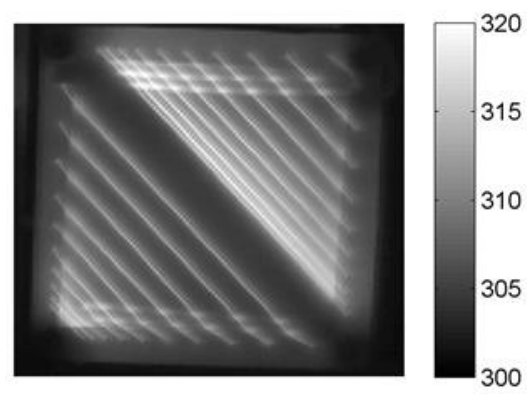

b) IBP REG

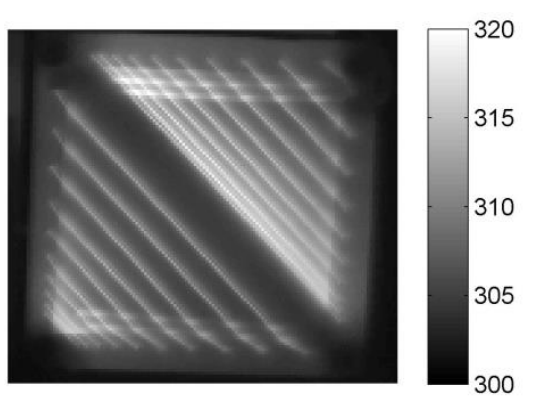

c) RS REG

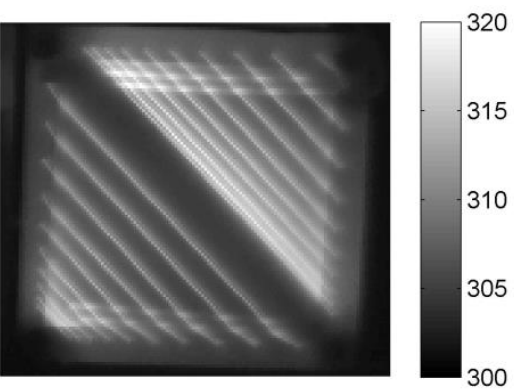

Fig. 6. Comparison of SR correction methods

Obtained SR images before and after correction were compared with original and interpolated thermograms. It can be noticed that interpolated thermogram and the IBP SR themogram with no correction applied are blurred (fig. 5). In LOC and REG methods details are emphasized and can be easily distinguished among each other. More detailed analysis revealed that temperatures in IBP HR images are mostly lower as it is in the original thermogram (fig. 6). Analysis of temperature profile (fig. 7) also led to the statement that narrow temperature slopes are flattened. It can be seen that corrected image is sharper than other ones.

a) Original

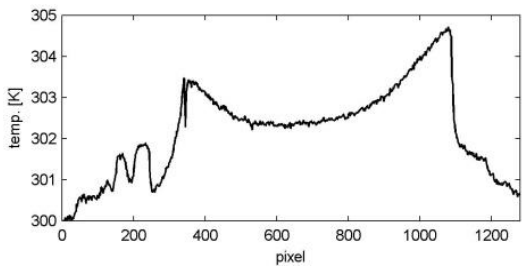

c) Interpolation REG

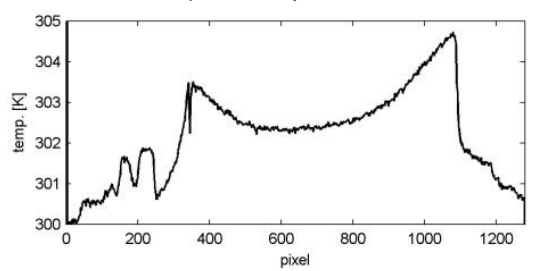

b) SR Interpolation without correction

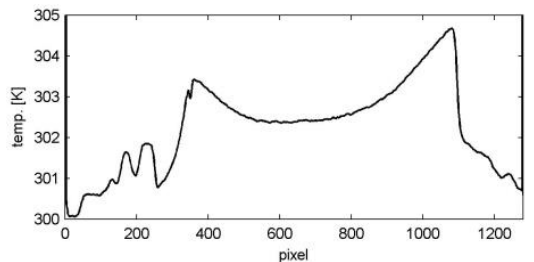

d) RS REG

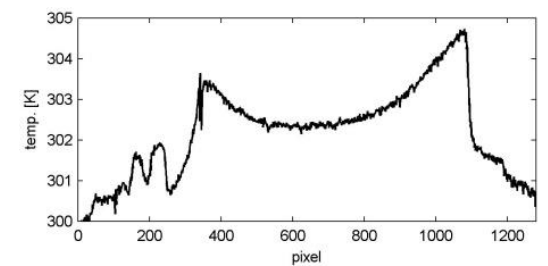

Fig. 7. Horizontal temperature profiles for original and SR thermograms

Analysis of horizontal temperature profile that was placed in the middle of image reveals, that interpolation smooths the line, so small disturbances are eliminated. Through the REG correction additional information is restored. Other SR methods (RS and IBP) are adding more informational content to the thermogram. Comparing profile and subjective visual assessment of the image, small fluctuations of temperature are influencing contrast. Through this small objects with low temperature increase over background are well separated and clearly visible.

Results of qualitative assessment are gathered in in table 1, table 2 and table 3. Comparision of SR images with original HR image it can be stated, that using SR original content structure is well retained. Differences are small, but in all cases original SR images are less similar to original HR image as corrected ones. More interesting is the analysis of IW-PSNR. Presence of noise in SR images is obvious. Nevertheless for the REG method, SR images are better denoised, what is presented in the significant increase of IW-PSNR and decrease od IW-MSE.

HISE and LAPE metrics are commonly used to estimate image focus. Thus blurred image of low quality will have lower score. In the HISE, the value of entropy corresponds to temperature distribution. When in region of interest image is sharp, then there is larger distribution of pixel values and entropy is higher. In the case of LAPE is used for analyzing high spatial frequencies associated with image border sharpness. Image clarity increases when LAPE is higher. In the case of complex scene with electrical equipment, application of correction procedure increases both HISE and LAPE. SR itself produces worse images as original, but correction process, especially with local method returns accuracy of temperature distribution. For laboratory stand results of SR are almost identical as the original. There is slight difference pointing at the fact, that SR images are clearer and sharper as the original. Deblurring was properly performed. 


\subsection{1/qirt.2016.046}

High difference of LAPE and HISE measures between interpolation and other SR methods are connected with the differences in the dynamic range of images. Lower informational content is bounded with high dynamic range of interpolated image (Fig. 7), which is significantly different even from the original image. Wide spread of pixel values is influencing the LAPE and HISE. According to that results of focus (blur) measures can't be compared between interpolation and other SR methods.

NIQE represents structure of image. Because the grayscale distribution is not corresponding with radiometric distribution, only local sharpness can be expressed by NIQE. For the real life images SR produces images with higher quality as original. For global correction methods, images are of the same quality as uncorrected SR images, for both IBP and RS methods.

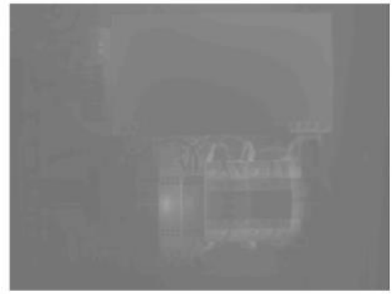

a)

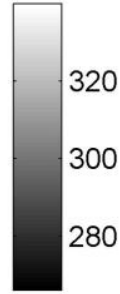

Fig. Dynamic range of SR images

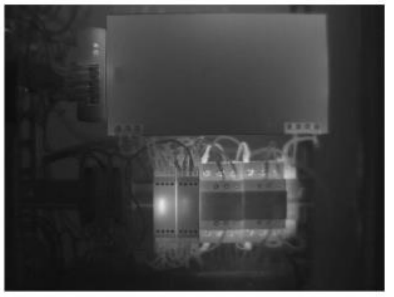

b)

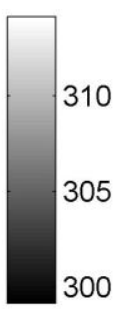

300

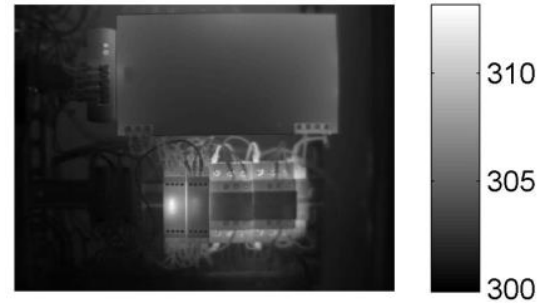

c)

Comparing LAPE and HISE for IBP and RS it can was found that regardless the test image or correction method IBP is slightly better than RS. It can be also found that local methods led to lower blurring than global methods. It can't be unanimously stated which of local correction methods is better. For electric equipment thermogram slightly better results are achieved with use of REG method, while for the lab stand LOC was generally a better choice.

SR images without correction have slightly lower IW-SSIM than corrected ones. It means that structure of original image is well preserved. IW-MSE analysis reveals that local correction methods allow generation of thermograms on high quality and low difference.

Table 1. Output image quality for IBP method

\begin{tabular}{|c|c|c|c|c|c|c|c|c|c|c|c|c|}
\hline \multirow{2}{*}{ measure } & \multicolumn{6}{|c|}{ Electric equipment } & \multicolumn{6}{|c|}{ Lab stand } \\
\hline & org. & SR & REG & LOC & GLOB1 & GLOB2 & org. & SR & REG & LOC & GLOB1 & GLOB2 \\
\hline $\begin{array}{l}\text { IW- } \\
\text { SSIM }\end{array}$ & 1.00 & 0.98 & 1.00 & 1.00 & 0.98 & 0.98 & 1.000 & 0.99 & 1.00 & 0.99 & 0.99 & 1.00 \\
\hline IW-MSE & $\begin{array}{l}0.00 \\
E+00\end{array}$ & $\begin{array}{l}8.36 \\
E-01\end{array}$ & $\begin{array}{l}1.64 \\
E-17\end{array}$ & $\begin{array}{l}6.68 \\
E-03\end{array}$ & $\begin{array}{l}8.18 \\
E-01\end{array}$ & $\begin{array}{l}8.18 \\
E-01\end{array}$ & $\begin{array}{l}0.00 \\
E+00\end{array}$ & $\begin{array}{l}2.16 \\
E-01\end{array}$ & $\begin{array}{l}2.27 \\
E-03\end{array}$ & $\begin{array}{l}1.98 \\
E-01\end{array}$ & $\begin{array}{l}1.99 \\
E-01\end{array}$ & $\begin{array}{l}8.09 \\
\text { E-18 }\end{array}$ \\
\hline $\begin{array}{l}\text { IW- } \\
\text { PSNR }\end{array}$ & 1000.0 & 48.9 & 215.9 & 69.8 & 49.0 & 49.0 & 1000.0 & 54.8 & 74.6 & 55.2 & 55.2 & 219.0 \\
\hline NIQE & 15.39 & 15.92 & 15.72 & 15.01 & 15.92 & 15.92 & 14.20 & 14.62 & 14.90 & 14.62 & 14.62 & 13.90 \\
\hline LAPE & 2.655 & 0.809 & 1.595 & 1.265 & 0.808 & 0.809 & 6.508 & 4.773 & 3.688 & 4.769 & 4.773 & 2.769 \\
\hline HISE & 0.096 & 0.184 & 0.201 & 0.188 & 0.184 & 0.184 & 0.389 & 0.774 & 0.633 & 0.773 & 0.774 & 0.304 \\
\hline
\end{tabular}

Table 2. Output image quality for RS method

\begin{tabular}{|c|c|c|c|c|c|c|c|c|c|c|c|c|}
\hline \multirow{2}{*}{ measure } & \multicolumn{6}{|c|}{ Electric equipment } & \multicolumn{6}{|c|}{ Lab stand } \\
\hline & org. & SR & REG & LOC & GLOB1 & GLOB2 & org. & SR & REG & LOC & GLOB1 & GLOB2 \\
\hline $\begin{array}{l}\text { IW- } \\
\text { SSIM }\end{array}$ & 1.00 & 0.99 & 1.00 & 1.00 & 0.99 & 0.99 & 1.00 & 0.99 & 1.00 & 1.00 & 0.99 & 0.99 \\
\hline IW-MSE & $\begin{array}{l}0.00 \\
E+00\end{array}$ & $\begin{array}{l}8.07 \\
E-01\end{array}$ & $\begin{array}{l}1.19 \\
E-17\end{array}$ & $\begin{array}{l}6.37 \\
E-03\end{array}$ & $\begin{array}{l}7.90 \\
E-01\end{array}$ & $\begin{array}{c}7.90 \text { E- } \\
01\end{array}$ & $\begin{array}{l}0.00 \\
E+00\end{array}$ & $\begin{array}{l}2.13 \\
E-01\end{array}$ & $\begin{array}{l}8.67 \\
E-18\end{array}$ & $\begin{array}{l}2.31 \\
E-03\end{array}$ & $\begin{array}{c}1.96 \text { E- } \\
01\end{array}$ & $\begin{array}{l}1.97 \\
E-01\end{array}$ \\
\hline $\begin{array}{l}\text { IW- } \\
\text { PSNR }\end{array}$ & 1000.0 & 49.0 & 217.3 & 70.1 & 49.1 & 49.1 & 1000.0 & 54.8 & 218.7 & 74.5 & 55.2 & 55.2 \\
\hline NIQE & 15.39 & 16.52 & 15.88 & 16.15 & 16.52 & 16.52 & 14.20 & 14.46 & 13.78 & 14.87 & 14.46 & 14.46 \\
\hline LAPE & 2.655 & 0.984 & 1.336 & 1.021 & 0.984 & 0.984 & 6.508 & 4.741 & 2.800 & 3.688 & 4.738 & 4.741 \\
\hline HISE & 0.096 & 0.158 & 0.180 & 0.152 & 0.158 & 0.158 & 0.389 & 0.767 & 0.312 & 0.631 & 0.766 & 0.767 \\
\hline
\end{tabular}

Comparing global correction with regional and local ones, it was found that hybrid approach, when known values remain unchanged and only one factor is applied to estimated thermopixles of HR termogram a good ratio between quality and computational load can be achieved. The possibility of generating better images than original ones 


\subsection{1/qirt.2016.046}

is offered by properties of SR techniques. Removing some part of distortions introduced by camera optics, aliasing effect, motion blur or other noise enhances thermograms, what is visible not only in subjective assessment but also in quantitative measeures.

Table 3. Output image quality for interpolation

\begin{tabular}{|c|c|c|c|c|c|c|c|c|c|c|c|c|}
\hline \multirow{2}{*}{ measure } & \multicolumn{6}{|c|}{ Electric equipment } & \multicolumn{6}{|c|}{ Lab stand } \\
\hline & org. & SR & REG & LOC & GLOB1 & GLOB2 & org. & SR & REG & LOC & GLOB1 & GLOB2 \\
\hline $\begin{array}{l}\text { IW- } \\
\text { SSIM }\end{array}$ & 1.00 & 0.98 & 1.00 & 1.00 & 0.98 & 0.98 & 1.00 & 0.99 & 1.00 & 1.00 & 0.99 & 0.99 \\
\hline \multirow{2}{*}{ IW-MSE } & 0.00 & 1.32 & 8.88 & 1.55 & 1.39 & 1.39 & 0.00 & 3.33 & 0.00 & 3.88 & 3.03 & 3.05 \\
\hline & $\mathrm{E}+0$ & $E+00$ & E-18 & $\mathrm{E}-02$ & $\mathrm{E}+00$ & $E+00$ & $E+00$ & $\mathrm{E}-01$ & $\mathrm{E}+00$ & $\mathrm{E}-03$ & E-01 & E-01 \\
\hline $\begin{array}{l}\text { IW- } \\
\text { PSNR }\end{array}$ & 1000.0 & 46.9 & 218.6 & 66.2 & 46.7 & 46.7 & 1000 & 52.9 & 1000.0 & 72.2 & 53.3 & 53.3 \\
\hline NIQE & 15.39 & 18.07 & 15.67 & 16.81 & 18.07 & 18.07 & 14.20 & 14.98 & 15.19 & 14.96 & 14.98 & 14.98 \\
\hline LAPE & 2.655 & 41.97 & 40.06 & 54.76 & 41.94 & 41.97 & 6.51 & 43.73 & 42.13 & 56.67 & 43.67 & 43.72 \\
\hline HISE & 0.096 & 7.793 & 5.094 & 7.849 & 7.788 & 7.793 & 0.389 & 7.784 & 5.140 & 7.863 & 7.774 & 7.784 \\
\hline
\end{tabular}

\section{Summary}

In the paper investigation concerning correction of temperatures in super-resolution thermograms was carried out. Application of global or local methods lead to obtainment of more reliable and sharper images with more certain temperatures as it is in the case of interpolated thermograms and those SR images with no correction applied. It was found that application of coefficients calculated locally is better than global approach. Local methods preserve dynamics in the image content. It offers also better adaptability to changes in structural content of thermogram, expressed in higher gradients of temperature. Obtained results confirmed validity of proposed approach. It was observed that interpolation of correction coefficients can generate additional noise, so regional approach, where in some neighbourhood same coefficient is applied can also be a reliable method.

Further research will be focused on the integration of radimetrical accuracy into the super resolution approach. Additional study is also needed for evaluation of proposed methods on wider group of examples. Also creation of new quality metrics, that will be suitable for measuring objectively the quality of infrared thermograms.

\section{REFERENCES}

[1] R. Y. Tsai, T. S. Huang, Multi-frame image restoration and registration, Adv. Comput. Vision Image Processing, Vol. 1(1984), pp. $317-339$

[2] H. Su, L. Tang, Y. Wu, et al. Spatially adaptive block-based super-resolution IEEE Transactions on Image Processing, 21 (2012), pp. 1031-1045 (TV)

[3] Y. Wang, R. Fevig and R. R. Schultz, Super-resolution mosaicking of UAV surveillance video, in 15th IEEE International Conference on Image Processing, 2008, pp. 345-348.

[4] C. Liu and D. Sun, A Bayesian approach to adaptive video super resolution, in 2011 IEEE Conference on Computer Vision and Pattern Recognition (CVPR), 2011, pp. 209-216.

[5] H. Greenspan, Super-resolution in medical imaging, The Computer Journal, 52 (2009), pp. 43-63

[6] D. Wallach, F. Lamare, G. Kontaxakis, et al. Super-resolution in respiratory synchronized positron emission tomography, IEEE Transactions on Medical Imaging, 31 (2012), pp. 438-448

[7] H. Zhang, Z. Yang, L. Zhang, et al. Super-resolution reconstruction for multi-angle remote sensing images considering resolution differences, Remote Sensing, 6 (2014), pp. 637-657

[8] M.T. Merino, J. Nunez, Super-Resolution of Remotely Sensed Images With Variable-Pixel Linear Reconstruction, IEEE Transactions on Geoscience and Remote Sensing, 45 (2007), pp. 1446-1457

[9] X. Wang, X. Tang, Hallucinating face by eigentransformation, IEEE Transactions on Systems, Man, and Cybernetics, Part C: Applications and Reviews, 35 (2005), pp. 425-434

[10] W. Jamrozik An example of surface fault estimation on the basis of super-resolution approach. Diagnostyka 2010 no 2, pp. 59-64

[11] https://www.testo.com/en/home/products/thermal_imaging/testothermalcameras.jsp

[12] http://www.fluke.com/fluke/sgen/infrared-camera/fluke-tix1000.htm?pid=78909

[13] http://www.infrared.avio.co.jp/en/products/ir-thermo/lineup/r300sr/spec.html

[14] Zomet, A.; Rav-Acha, A.; Peleg, S., "Robust super-resolution". Computer Vision and Pattern Recognition, 2001. CVPR 2001. Proceedings of the 2001 IEEE Computer Society, vol.1 (2001), pp. 645-650

[15] Irani M., Peleg Sh., "Improving resolution by image registration". Journal CVGIP: Graphical Models and Image Processing archive, Vol. 53 (1991), pp.231-239 


\subsection{1/qirt.2016.046}

[16] Panagiotopoulou A., Anastassopoulos V., "Super-Resolution Reconstruction of Thermal Infrared Images". Proceedings of the 4th WSEAS International Conference on REMOTE SENSING (REMOTE'08) (2008), pp. 40-44

[17] Y. Sun, S. Duthaler, B.J. Nelson, Autofocusing in computer microscopy: selecting the optimal focus algorithm, Microscopy Research and Technique 65 (2004) 139-149

[18] N.K. Chern, P.A. Neow, M.H. Ang, Practical issues in pixel-based autofocusing for machine vision, in: Proceedings of the International Conference on Robotics and Automation, vol. 3, 2001, pp. 2791-2796

[19] A. Mittal, R. Soundararajan and A. C. Bovik, " Making a Completely Blind Image Quality Analyzer ", IEEE Signal processing Letters, pp. 209-212, vol. 22, no. 3, March 2013

[20] Zhou Wang and Qiang Li, "Information Content Weighting for Perceptual Image Quality Assessment," IEEE Transactions on Image Processing, vol. 20, no. 5, pp. 1185-1198, May 2011 